Abstract S58 Table 1 Measured values at baseline and after 6 months' treatment.

\begin{tabular}{llll}
\hline & Baseline & 6 months & Difference \\
\hline ppFEV1 $^{1}$ & $24.8(7.13)$ & $33.4(10.33)$ & $8.6^{3}$ \\
BMI $^{1}$ & $21.3(4.13)$ & $23.9(4.29)$ & $2.6^{3}$ \\
RSI $^{2}$ & $15(10.75-23)$ & $5(2.25-7)$ & $10^{3}$ \\
HARQ $^{2}$ & $26.5(16-39)$ & $7(3.75-12.25)$ & $19.5^{3}$ \\
SNOT-20 $^{2}$ & $36.5(22-42)$ & $20(10-31.25)$ & $16.5^{3}$ \\
\hline
\end{tabular}

${ }^{1}$ mean (Standard Deviation)

2 median (IQR)

${ }^{3} \mathrm{p}<0.001$

ppFEV1 - percentage predicted Forced Expiratory Volume in 1 second, BMI - Body Mass Index, RSI - Reflux Symptom Index, HARQ - Hull Airway Reflux Questionnaire, SNOT-20 Sino-Nasal Outcome Test 20

Hull Airway Reflux Questionnaire (HARQ) as patient-reported outcome measures (PROMs) to assess the effect of triple CFTR modulators on sino-nasal and laryngoesophageal reflux symptoms. Questionnaires, lung function and weight were recorded at baseline before starting treatment and after 6 months on treatment.

Results 32 patients (23 male) starting elexacaftor/tezacaftor/ivacaftor were studied. Their baseline characteristics were mean age 34.3 (range 20-65) years, $\mathrm{FEV}_{1} \%$ predicted 24.8 (11-40), weight $63.2 \mathrm{~kg}(35-99.8)$ and BMI $21.28 \mathrm{~kg} / \mathrm{m}^{2}$ (13.2-31.1). All patients continued with treatment throughout the study period. At 6 months there was an improvement in mean FEV1\% predicted of 8.63 and BMI $2.6 \mathrm{~kg} / \mathrm{m}^{2}$. Patient reported outcome measures showed significant improvement (table 1): median scores RSI 10, HARQ 19.5 and SNOT 16 $(\mathrm{p}<0.001$ for all outcomes).

Discussion This study shows significant improvement in lung function, weight and sino-nasal and laryngopharyngeal reflux PROMs in patients with advanced CF. The SNOT-20, RSI and HARQ scores showed improvement that exceeded recognised clinically significant changes in these metrics.

\section{S59 ADHERENCE TO NEBULISED THERAPIES IN PEOPLE WITH CYSTIC FIBROSIS STARTING ELEXACAFTOR/ TEZACAFTOR/IVACAFTOR (KAFTRIO)}

IB Howell, A Tugwell, D Bhaskaran, NJ Bell. Bristol Adult Cystic Fibrosis Centre, Bristol, UK

\subsection{6/thorax-2021-BTSabstracts.65}

Introduction Cystic Fibrosis HealthHub (CFHH) is a digital platform in use by 17 adult CF centres in the UK which improves patient self-care by objectively monitoring adherence to nebulised therapies delivered via e-Track nebulisers which record device usage on a central server. This study aimed to objectively measure a perceived decrease in adherence with nebulised therapies following initiation of the Elexacaftor-Tezacaftor-Ivacaftor oral CFTR modulator therapy.

Methods We identified all patients on Kaftrio currently enrolled in $\mathrm{CFHH}$ who regularly uploaded data. We compared average CFHH-measured adherence 3 months before and after starting Kaftrio. We reviewed documentation on our clinical database of any patient or healthcare professional decision to change nebuliser usage.

Results 154 patients were taking Kaftrio. 71 were not enrolled in $\mathrm{CFHH}, 34$ did not regularly upload data, and 2 had been instructed to change therapy during the study period leaving

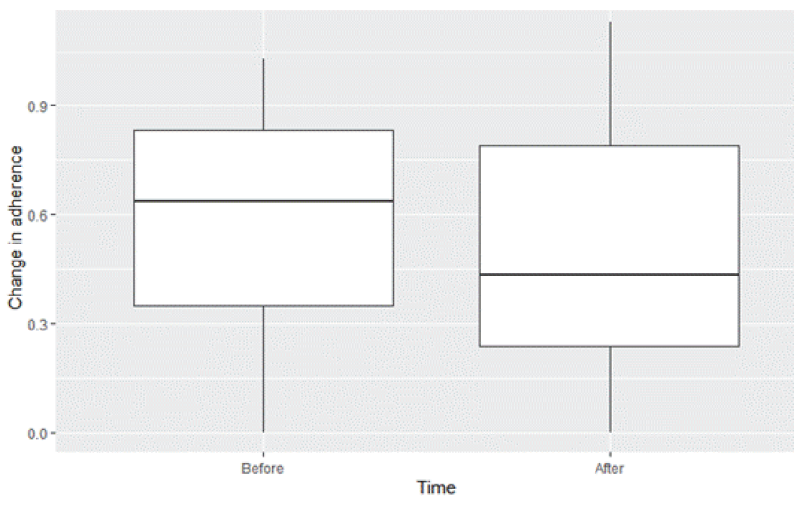

Abstract S59 Figure 1 Box plot comparing average adherence of nebulised therapy before and after Kaftrio initition

47 patients included in analysis. 31 patients (65\%) reduced their adherence to nebulised therapies following Kaftrio use. Median nebulised therapy adherence dropped from $65 \%$ to $42 \%(\mathrm{p}<0.003$, Wilcoxson Signed Rank) pre and post Kaftrio introduction respectively (figure 1 ). Of the 47 patients, 28 (60\%) communicated a decision to change therapy with the CF team, while 19 (40\%) did not communicate this change.

Discussion Our data demonstrates a reduction in nebulised therapy adherence after Kaftrio initiation. Decisions to reduce adherence were often patient driven and not disclosed to clinicians.

Our findings underline the importance of including objective measures of adherence to inhaled therapies in the design of CFTR modulator studies.

The lack of CFHH uploads for 34 patients highlights the challenges in monitoring adherence in clinical practice; in our experience, these patients were less adherent to treatment.

We plan to conduct a qualitative study to explore factors influencing patient decisions to stop or continue medication.

\section{S60 OBSERVATIONAL STUDY OF IVACAFTOR IN PEOPLE WITH CYSTIC FIBROSIS AND SELECTED NON-G551D GATING MUTATIONS: FINAL RESULTS FROM VOCAL}

${ }^{1} \mathrm{NJ}$ Simmonds, ${ }^{2} \mathrm{~K}$ van der Ent, ${ }^{3} \mathrm{C}$ Colombo, ${ }^{4} \mathrm{~N}$ Kinnman, ${ }^{4} \mathrm{C}$ DeSouza, ${ }^{4} \mathrm{~T}$ Thorat, ${ }^{4} \mathrm{~K}$ Chandarana, ${ }^{5} \mathrm{C}$ Castellani. ${ }^{1}$ Adult Cystic Fibrosis Centre, Royal Brompton Hospital and Imperial College London, London, UK; ${ }^{2}$ Department of Pediatric Respiratory Diseases, University Medical Center Utrecht, Utrecht, The Netherlands; ${ }^{3}$ Fondazione IRCCS Ca' Granda Ospedale Maggiore Policlinico, University of Milan, Milan, Italy; ${ }^{4}$ Vertex Pharmaceuticals Incorporated, Boston, USA; ${ }^{5}$ Cystic Fibrosis Centre, IRCCS Istituto Giannina Gaslini, Genoa, Italy

\subsection{6/thorax-2021-BTSabstracts.66}

Introduction and Objectives VOCAL, a Phase 4 observational study (NCT02445053), assessed real-world effectiveness of ivacaftor (IVA) in people with cystic fibrosis (pwCF) with $\geq 1$ non-G551D gating mutation (G178R, S549N, S549R, G551S, G1244E, S1251N, S1255P or G1349D).

Methods PwCF aged $\geq 6$ years in Italy, the Netherlands and the UK who were IVA-naïve or on IVA for $\leq 18$ months at enrolment were eligible. Data were recorded for 12 months pre-IVA and up to 48 months after enrolment. Continuous outcomes (e.g. percent predicted forced expiratory volume in 1 second $\left[\mathrm{ppFEV}_{1}\right]$, body mass index $[\mathrm{BMI}]$ ) were assessed from baseline (the last pre-IVA value recorded) in 6-month intervals up to 48 months post-IVA using a mixed model for 
repeated measures; total pulmonary exacerbations (PEx) and healthcare resource utilisation (HCRU) post- vs pre-IVA were assessed using a negative binomial model.

Results 65 of 73 (89\%) completed the study; mean IVA exposure was 49.5 months (range, 2-64). Mean baseline age was 26.9 years (standard deviation [SD], 13.5). Mean baseline $\operatorname{ppFEV}_{1}(64.83$ [SD, 23.61]) increased by a least-squares (LS) mean of 10.77 (standard error [SE], 1.28) within 6 months that was sustained up to 48 months (10.27 [SE, 1.45]). Mean baseline BMI (pwCF $\geq 20$ years, $\mathrm{n}=49 ; 22.95 \mathrm{~kg} / \mathrm{m}^{2} \quad[\mathrm{SD}$, 3.81]) increased by an LS mean of 0.79 (SE, 0.14) within 6 months and $1.30(\mathrm{SE}, 0.24)$ at 48 months. Mean baseline BMI $z$ score (pwCF $<20$ years, $\mathrm{n}=24 ;-0.41$ [SD, 0.89]) increased by an LS mean of 0.54 (SE, 0.11) within 6 months and 0.41 (SE, 0.14) at 48 months. Estimated annualised rates of PEx, PEx requiring hospitalisation, all-cause hospitalisation and PEx requiring acute antibiotics decreased by $>50 \%$ in the first 12 months post- vs 12 months pre-IVA, and changes were sustained during treatment. No new safety concerns were identified.

Conclusions IVA showed sustained effectiveness in clinical outcomes and decreased HCRU.

Please refer to page A189 for declarations of interest related to this abstract.

\section{S61 RESPIRATORY MICROBIOLOGY OUTCOMES FROM AN OBSERVATIONAL STUDY OF IVACAFTOR IN PEOPLE WITH CYSTIC FIBROSIS AND NON-G551D GATING MUTATIONS (VOCAL)}

${ }^{1} \mathrm{C}$ Castellani, ${ }^{2} \mathrm{NJ}$ Simmonds, ${ }^{3} \mathrm{C}$ Colombo, ${ }^{4} \mathrm{~N}$ Kinnman, ${ }^{4} \mathrm{C}$ DeSouza, ${ }^{4} \mathrm{~T}$ Thorat, ${ }^{4} \mathrm{M}$ Chew, ${ }^{4} \mathrm{~K}$ Chandarana, ${ }^{5} \mathrm{~K}$ van der Ent. ${ }^{1}$ Cystic Fibrosis Centre, IRCCS Istituto Giannina Gaslini, Genoa, Italy; ${ }^{2}$ Adult Cystic Fibrosis Centre, Royal Brompton Hospital and Imperial College London, London, UK; ${ }^{3}$ Fondazione IRCCS Ca' Granda Ospedale Maggiore Policlinico, University of Milan, Milan, Italy; ${ }^{4}$ Vertex Pharmaceuticals Incorporated, Boston, USA; ${ }^{5}$ Department of Pediatric Respiratory Diseases, University Medical Center Utrecht, Utrecht, The Netherlands

\subsection{6/thorax-2021-BTSabstracts.67}

Introduction and Objectives Certain respiratory pathogens are associated with reduced lung function and disease progression in people with cystic fibrosis (pwCF). We report respiratory microbiology results from a Phase 4 observational study (NCT02445053) assessing real-world effectiveness of ivacaftor (IVA) in pwCF with non-G551D gating mutations (G178R, S549N, S549R, G551S, G1244E, S1251N, S1255P or G1349D). Methods PwCF aged $\geq 6$ years in Italy, the Netherlands and the UK who were IVA-naïve or on IVA for $\leq 18$ months at enrolment were eligible. Data were recorded for 12 months pre-IVA and up to 48 months after enrolment. Microbiology cultures were taken via sputum, throat or oropharyngeal swabs. Results 65 of 73 (89\%) completed the study; mean IVA exposure was 49.5 months (range, 2-64). Mean (standard deviation) baseline age and percent predicted forced expiratory volume in 1 second were 26.9 (13.5) years and 64.83 (23.61), respectively. In the 12 months pre-IVA, 279 cultures were obtained from $69 \mathrm{pwCF}$ and 182 cultures in $64 \mathrm{pwCF}$ at year 4 following IVA treatment. Prevalence of $P$. aeruginosa, A. fumigatus and S. maltophilia was $55.1 \%, 30.4 \%$ and $11.6 \%$, respectively, in the 12 months pre-IVA and was reduced to $52.9 \%, 18.6 \%$ and $7.1 \%$ in year 1 and $41.5 \%$, $16.9 \%$ and $4.6 \%$ in year 2 on IVA. Sustained or further reductions were observed through 48 months of treatment.
Prevalence of other pathogens was variable or too low to evaluate. $70 \%$ of pwCF were on chronic oral and/or inhaled antibiotics pre-IVA vs $68 \%$ at 48 months. Use of other chronic inhaled therapies was stable throughout the study.

Conclusions Lower prevalence of $P$. aeruginosa, A. fumigatus and S. maltophilia was observed with prolonged IVA treatment for up to 48 months in real-world settings. Chronic medication use remained stable.

Please refer to page A189 for declarations of interest related to this abstract.

\section{S62 THE MICROBIOLOGY OF BRONCHIECTASIS EXACERBATIONS IN THE UK EMBARC REGISTRY AND IMPLICATIONS FOR PRESCRIBING IN PRIMARY CARE: A COHORT STUDY}

${ }^{1} \mathrm{~F}$ Mosgrove, ${ }^{2} \mathrm{C}$ Haworth, ${ }^{2} \mathrm{M}$ Loebinger, ${ }^{3} \mathrm{M}$ Crichton, ${ }^{4} \mathrm{P}$ Goeminne, ${ }^{3} \mathrm{~A}$ Shoemark, ${ }^{5}$ E Polverino, ${ }^{6} \mathrm{~S}$ Aliberti, ${ }^{7} \mathrm{~A}$ DeSoyza, ${ }^{3} \mathrm{JD}$ Chalmers. ${ }^{1}$ University of Aberdeen, Aberdeen, UK; ${ }^{2}$ The Royal Papworth Hospital, Cambridge, UK; ${ }^{3}$ University of Dundee, Dundee, UKi ${ }^{4}$ University of Leuven, Leuven, Belgium; ${ }^{5}$ Hospital Universitari Vall d, Barcelona, Spain; ${ }^{6}$ University of Milan, Milan, Italy; ${ }^{7}$ Newcastle University, Newcastle, UK

\subsection{6/thorax-2021-BTSabstracts.68}

Introduction The British Thoracic Society (BTS) guidelines for the management of Bronchiectasis advise that sputum samples are sent for microbiology at baseline and at exacerbation. Guidelines recommend that antibiotic treatment at exacerbations should be guided by previous sputum microbiology. Amoxicillin and Doxycycline are guideline recommended empirical choices in primary care where no prior microbiology is available.

Methods We aimed to examine the UK cohort of the European Multicentre Bronchiectasis Registry (EMBARC) to determine whether management of these patients was in line with BTS guideline recommendations and examine antibiotic sensitivities at exacerbation. The organisms grown were identified and their sensitivity to amoxicillin and doxycycline, using sputum culture sensitivity data, was defined.

Results 7931 UK patients were analysed. 53.3\% of patients had sputum sent at baseline and of those with exacerbations $42.3 \%$ had a sputum sample sent at exacerbation within 1 year of baseline. $21.7 \%$ of exacerbating patients had a prior stable sputum result available to guide exacerbation prescribing with only $34.8 \%$ of these showing concordance, with baseline sputum microbiology predictive of exacerbation sputum microbiology. Haemophilus influenzae (25.7\%) and Pseudomonas aeruginosa $(19.8 \%)$ are the most common organisms grown at exacerbation. Examination of all organisms grown at first exacerbation shows that $36.2 \%$ of these are susceptible to amoxicillin and $55.6 \%$ are susceptible to doxycycline. The difference in susceptibility between amoxicillin and doxycycline was largely accounted for by beta-lactamase producing H.influenzae, Moraxella catarrhalis and Staphylococcus aureus. Excluding P. aeruginosa gives $47.2 \%$ coverage of remaining organisms by amoxicillin and 73\% coverage with doxycycline. Conclusion Concordance with BTS guidelines on management of Bronchiectasis is low with sputum samples sent infrequently in stable state and at exacerbation. Microbiology concordance between stable and exacerbation samples is poor. There are high levels of innate and acquired resistance to amoxicillin making doxycycline potentially a more effective first choice antibiotic where no sputum culture results are available to guide prescribing. 